\title{
MULTIPLICITY RESULTS TO A CLASS OF VARIATIONAL-HEMIVARIATIONAL INEQUALITIES
}

\author{
GABRIELE BONANNO AND PATRICK WINKERT
}

\begin{abstract}
This paper deals with variational-hemivariational inequalities involving the $p$-Laplace operator and a nonlinear Neumann boundary condition. Based on an abstract critical point result, which is developed at the beginning of the paper, it is shown the existence of at least three solutions to such inequalities whereby the cases $p>N$ and $p \leq N$ are treated separately. The applicability of these results is emphasized with suitable examples.
\end{abstract}

\section{INTRODUCTION}

Let $\Omega$ be a non-empty, bounded, open subset of the real Euclidian space $\mathbb{R}^{N}$, $N \geq 1$, with $C^{1}$-boundary $\Gamma:=\partial \Omega$ and let $q \in L^{\infty}(\Omega)$ satisfying $q \geq 0, q \not \equiv 0$. We consider the following problem: Find $u \in K$ such that, for all $v \in K$,

$$
\begin{aligned}
& \int_{\Omega}|\nabla u(x)|^{p-2} \nabla u(x) \cdot \nabla(v(x)-u(x)) d x \\
& \quad+\int_{\Omega} q(x)|u(x)|^{p-2} u(x)(v(x)-u(x)) d x \\
& \quad+\int_{\Omega} \lambda \alpha(x) F^{\circ}(u(x) ; v(x)-u(x)) d x \\
& \quad+\int_{\Gamma} \mu \beta(x) G^{\circ}(\gamma u(x) ; \gamma v(x)-\gamma u(x)) d \sigma \geq 0,
\end{aligned}
$$

where $K$ is a closed convex subset of the usual Sobolev space $W^{1, p}(\Omega), 1<p<\infty$, containing the zero function while $\alpha \in L^{1}(\Omega), \beta \in L^{1}(\Gamma)$, fulfill $\alpha(x) \geq 0$ for a.a. $x \in \Omega, \alpha \neq \equiv$ and $\beta(x) \geq 0$ for a.a. $x \in \Gamma$. The values $\lambda$ and $\mu$ are real parameters with $\lambda>0$ and $\mu \geq 0$ specified later. By $F^{\circ}$ and $G^{\circ}$ we denote Clarke's generalized directional derivatives of the locally Lipschitz continuous functions $F, G: \mathbb{R} \rightarrow \mathbb{R}$ given in the form

$$
F(\xi)=\int_{0}^{\xi} f(t) d t, \quad G(\xi)=\int_{0}^{\xi} g(t) d t
$$

with locally essentially bounded functions $f, g: \mathbb{R} \rightarrow \mathbb{R}$. As usual, we denote by $\gamma: W^{1, p}(\Omega) \rightarrow L^{p}(\Gamma)$ the well-known trace operator being linear and compact.

The aim of this paper is to provide multiplicity results to inequality (1.1). We present different existence theorems showing the existence of at least three distinct solutions of (1.1) provided the number $\lambda$ belongs to a specific interval and the parameter $\mu$ is sufficiently small. Since $C(\bar{\Omega})$ is compactly embedded into $W^{1, p}(\Omega)$

2010 Mathematics Subject Classification. 35J87, 49J40, 49J52, 49J53.

Key words and phrases. Elliptic variational-hemivariational inequality, Nonsmooth critical point theory, $p$-Laplacian. 
when $p>N$, the various cases $p \leq N$ and $p>N$ are discussed separately with different assumptions on the data $f$ and $g$. Our main results are stated in Theorem $3.1(p>N$, see Section 3) and Theorem $4.1(p \leq N$, see Section 4). The main idea in the proofs is the usage of an appropriate abstract three-critical-point-result for non-smooth functionals which is proved in Section 2 based on results in [8].

Existence and multiplicity results for variational-hemivariational inequalities have been obtained under different structure and regularity conditions on the nonlinear functions by various authors. We refer, for example, to [4], [23], [24], [28] and [36] as well as the references therein. It is clear that problem (1.1) becomes a hemivariational inequality if $K$ coincides with the whole space $W^{1, p}(\Omega)$. Such inequalities have been handled for example in [2], [3], [13], [15], [29], [34] and [35]. In the context of infinitely many solutions to (1.1) we refer to the recent results stated in [9] and [41] for $p>N$ while existence results to (1.1) via the method of sub- and supersolution can be found in [14] and [16].

Let us comment on some relevant special cases of (1.1).

(A) If $K=W^{1, p}(\Omega)$ and $F, G$ are smooth, problem (1.1) reduces to

$$
\begin{aligned}
& \int_{\Omega}|\nabla u(x)|^{p-2} \nabla u(x) \cdot \nabla \varphi(x) d x+\int_{\Omega} q(x)|u(x)|^{p-2} u(x) \varphi(x) d x \\
& +\int_{\Omega} \lambda \alpha(x) F^{\prime}(u(x)) \varphi(x) d x+\int_{\Gamma} \mu \beta(x) G^{\prime}(\gamma u(x)) \gamma \varphi(x) d \sigma=0,
\end{aligned}
$$

for all $\varphi \in W^{1, p}(\Omega)$ which means that $u \in W^{1, p}(\Omega)$ is the weak solution of the nonlinear boundary value problem

$$
\begin{aligned}
& -\Delta_{p} u(x)+q(x)|u(x)|^{p-2} u(x)+\lambda \alpha(x) F^{\prime}(u(x))=0 \quad \text { in } \Omega, \\
& \frac{\partial u}{\partial \nu}(x)+\mu \beta(x) G^{\prime}(\gamma u(x))=0 \quad \text { on } \Gamma,
\end{aligned}
$$

where $(\partial u / \partial \nu)(x)=|\nabla u|^{p-2}(\partial u / \partial n)(x)$ with $(\partial u / \partial n)(x)$ being the outer normal derivative of $u$ at $x \in \Gamma$. Regarding existence and multiplicity of solutions to (1.2) we point out (without guarantee of completeness) the papers in [20], [21], [22], [30], [32], [37], [46], [48], and the references therein. Referring to homogeneous Neumann problems, the existence of at least three solutions in case $p>N$ was shown with different methods for example in [1], [5] and [6] (see also [7] for infinitely many solutions) while the more complicated case $p \leq N$ was recently studied in [19].

(B) In case $f=g=0,(1.1)$ is a classical variational inequality of the form

$$
\begin{aligned}
u \in K: \quad \int_{\Omega}|\nabla u(x)|^{p-2} \nabla u(x) \cdot \nabla(v(x)-u(x)) d x & \\
& +\int_{\Omega} q(x)|u(x)|^{p-2} u(x)(v(x)-u(x)) d x \geq 0, \quad \forall v \in K,
\end{aligned}
$$

whose treatment is well-known (see for example the monograph of Kinderlehrer in [25]).

(C) As mentioned above, problem (1.1) reduces to a so-called hemivariational inequality provided $K=W^{1, p}(\Omega)$. This contains as a special case the 
subsequent elliptic inclusion

$$
\begin{aligned}
-\Delta_{p} u(x)+q(x)|u(x)|^{p-2} u(x)+\lambda \alpha(x) \partial F(u(x)) \ni 0 & \text { in } \Omega, \\
\frac{\partial u}{\partial \nu}(x)+\mu \beta(x) \partial G(\gamma u(x)) \ni 0 & \text { on } \Gamma,
\end{aligned}
$$

whereby the multivalued functions $\partial F, \partial G$ stand for Clarke's generalized gradient (see Section 2 for more details). Concerning multiple solutions such inclusions have been studied in [31] and [47]. Regarding the existence

of infinitely many solutions for homogeneous problems of type (1.3) we also mention the paper of Candito [11] and the work of Kristály-Motreanu (see [27]) where in the second paper the authors do not require that $W^{1, p}(\Omega)$ is continuously embedded into $C(\bar{\Omega})$. Likewise, we draw attention to a paper of Kristály-Moroşanu in which a new competition phenomena between oscillatory and pure power terms has been described (cf. [26]).

It should be noted that our variational-hemivariational inequality is equivalent to the multi-valued variational inequality

$$
u \in K:\left\{\begin{array}{l}
\eta(x) \in \partial F(u(x)) \text { a.e. in } \Omega, \xi(x) \in \partial G(\gamma u(x)) \text { a.e. in } \Gamma, \\
\int_{\Omega}|\nabla u(x)|^{p-2} \nabla u(x) \cdot \nabla(v(x)-u(x)) d x \\
\quad+\int_{\Omega} q(x)|u(x)|^{p-2} u(x)(v(x)-u(x)) d x \\
\quad+\int_{\Omega} \lambda \alpha(x) \eta(x)(v(x)-u(x)) d x \\
\quad+\int_{\Gamma} \mu \beta(x) \xi(x)(\gamma v(x)-\gamma u(x)) d \sigma \geq 0, \quad \forall v \in K,
\end{array}\right.
$$

provided the elements of $\partial F, \partial G$ fulfill a suitable growth condition and $K$ has lattice structure, which means, if $u, v \in K$, then $\max \{u, v\}, \min \{u, v\} \in K$. In other words, $u$ is a solution of (1.1) if and only if $u$ is a solution of (1.4). This interesting result was recently published in [12].

We also would like to mention the recent work in [44] and references therein concerning three critical points theorems involving smooth functionals. For more information about (variational-)hemivariational inequalities we refer the reader to the monographs in [42] and [43].

\section{Preliminaries}

Let us recall some basic facts on non-smooth analysis which we will need in later considerations. Let $(X,\|\cdot\|)$ be a real Banach space and denote by $X^{*}$ its dual space while the duality pairing between $X$ and $X^{*}$ is denoted by $\langle\cdot, \cdot\rangle$. A function $f: X \rightarrow \mathbb{R}$ is said to be locally Lipschitz continuous if for every $x \in X$ there exist a neighborhood $U_{x}$ of $x$ and a constant $L_{x} \geq 0$ such that

$$
|f(y)-f(z)| \leq L_{x}\|y-z\|, \quad \forall y, z \in U_{x} .
$$

The term $f^{\circ}(x ; y), x, y \in X$ stands for the generalized directional derivative of $f$ at the point $x$ along the direction $y$ which is given by

$$
f^{\circ}(x ; y):=\limsup _{z \rightarrow x, t \rightarrow 0^{+}} \frac{f(z+t y)-f(z)}{t},
$$

(see [18, Chapter 2]). Let $f_{1}, f_{2}: X \rightarrow \mathbb{R}$ be locally Lipschitz continuous functions. Then we have

$$
\left(f_{1}+f_{2}\right)^{\circ}(x ; y) \leq f_{1}^{\circ}(x ; y)+f_{2}^{\circ}(x ; y), \quad \forall x, y \in X .
$$


The generalized gradient of a locally Lipschitz continuous function $f$ at $x$, denoted by $\partial f(x)$, is the set

$$
\partial f(x):=\left\{x^{*} \in X^{*}:\left\langle x^{*}, y\right\rangle \leq f^{\circ}(x ; y), \forall y \in X\right\} .
$$

An element $x \in X$ is a (generalized) critical point of $f$ if it satisfies the condition

$$
f^{\circ}(x ; y) \geq 0, \quad \forall y \in X,
$$

or equivalently, $0 \in \partial f(x)$ (see [17]).

Let $I: X \rightarrow]-\infty,+\infty]$ be a non-smooth function represented as a sum of a locally Lipschitz continuous function $f: X \rightarrow \mathbb{R}$ and a convex, proper and lower semicontinuous function $j: X \rightarrow]-\infty,+\infty]$, that is $I:=f+j$. An element $u \in X$ is a (generalized) critical point of $I$ if

$$
f^{\circ}(u ; v-u)+j(v)-j(u) \geq 0, \quad \forall v \in X
$$

is satisfied (see [39, Chapter 3] and [40]).

Now, let us give the assumptions concerning our first result. For a reflexive Banach space $X$, the functional $\Phi: X \rightarrow \mathbb{R}$ is assumed to be sequentially weakly lower semicontinuous and coercive while $\Upsilon: X \rightarrow \mathbb{R}$ is supposed to be sequentially weakly upper semicontinuous. By $\lambda$ we denote a positive real parameter, $j: X \rightarrow$ ]$-\infty,+\infty]$ is a convex, proper and lower semicontinuous functional and $D(j)$ stands for the effective domain of $j$. Then we define

$$
\Psi:=\Upsilon-j \quad \text { and } \quad J_{\lambda}:=\Phi-\lambda \Psi=(\Phi-\lambda \Upsilon)+\lambda j:=\Theta_{\lambda}+\lambda j,
$$

while it is supposed that

$$
D(j) \cap \Phi^{-1}(]-\infty, r[) \neq \emptyset, \quad \text { for all } r>\inf _{X} \Phi .
$$

Now we define

$$
\varphi_{1}(r)=\inf _{y \in \Phi^{-1}(]-\infty, r[)} \frac{\left(\sup _{x \in \Phi^{-1}(]-\infty, r[)} \Psi(x)\right)-\Psi(y)}{r-\Phi(y)}, \quad \text { for all } r>\inf _{X} \Phi,
$$

respectively,

$$
\varphi_{2}(r)=\sup _{y \in \Phi^{-1}(] r,+\infty[)} \frac{\Psi(y)-\left(\sup _{\left.\left.x \in \Phi^{-1}(]-\infty, r\right]\right)} \Psi(x)\right)}{\Phi(y)-r}, \quad \text { for all } r<\sup _{X} \Phi .
$$

We have the following result.

Theorem 2.1. Assume that there is $r \in] \inf _{X} \Phi, \sup _{X} \Phi\left[\right.$ such that $\varphi_{1}(r)<\varphi_{2}(r)$. Further suppose that the functional $J_{\lambda}$ is bounded from below and satisfies the (PS)condition for each $\lambda \in \Lambda:=] \frac{1}{\varphi_{2}(r)}, \frac{1}{\varphi_{1}(r)}\left[\right.$. Then, for each $\lambda \in \Lambda, J_{\lambda}$ has three distinct critical points.

Proof. First, we observe that, thanks to [40, Corollary 1.3], $J_{\lambda}$ is coercive. Now, we want to show that $J_{\lambda}$ has a local minima $u_{1} \in \Phi^{-1}(]-\infty, r[)$ and a local minima $u_{2} \in \Phi^{-1}(] r,+\infty[)$. Let $\lambda \in \Lambda$ be fixed. We are going to show that there is $u_{1} \in D(j) \cap \Phi^{-1}(]-\infty, r[)$ such that $J_{\lambda}\left(u_{1}\right) \leq J_{\lambda}(u)$ for all $u \in \Phi^{-1}(]-\infty, r[)$. Taking into account $\frac{1}{\lambda}>\varphi_{1}(r)$, we find $\bar{u} \in D(j)$ such that $\Phi(\bar{u})<r$ and $J_{\lambda}(\bar{u})=$ $\Phi(\bar{u})-\lambda \Psi(\bar{u})<r-\lambda \sup _{\Phi(x)<r} \Psi(x)$. Putting $L:=\frac{r-\Phi(\bar{u})}{\lambda}+\Psi(\bar{u})$ yields

$$
\sup _{\Phi(x)<r} \Psi(x)<L \text {. }
$$


Let us take

$$
\Psi_{L}(u)=\min \{\Psi(u), L\} .
$$

As $j$ is sequentially weakly lower semicontinuous (see [10, Corollary III.8]), we can easily prove that $\Psi_{L}$ is sequentially weakly upper semicontinuous. We take $J=\Phi-\lambda \Psi_{L}$ and note that $J$ is sequentially weakly lower semicontinuous and coercive which guarantees that its global minimum, namely $u_{0}$, exists (see [45, Theorem 1.2]). If $J\left(u_{0}\right)=J(\bar{u})$, we put $u_{1}=\bar{u}$, so $u_{1} \in \Phi^{-1}(]-\infty, r[)$ is a local minima of $J_{\lambda}$. Let us consider the case $J\left(u_{0}\right)<J(\bar{u})$. Then, we obtain

$$
\Phi\left(u_{0}\right)-\lambda \Psi_{L}\left(u_{0}\right)<\Phi(\bar{u})-\lambda \Psi_{L}(\bar{u}),
$$

thus

$$
\Phi\left(u_{0}\right)<\lambda \Psi_{L}\left(u_{0}\right)+\Phi(\bar{u})-\lambda \Psi_{L}(\bar{u}) \leq \lambda L+\Phi(\bar{u})-\lambda \Psi(\bar{u})=r .
$$

Thanks to $(2.3)$ we see at once that $\Psi\left(u_{0}\right)<L$. Hence, it follows

$$
\Phi\left(u_{0}\right)-\lambda \Psi\left(u_{0}\right)=\Phi\left(u_{0}\right)-\lambda \Psi_{L}\left(u_{0}\right) \leq \Phi(u)-\lambda \Psi_{L}(u), \quad \forall u \in X .
$$

With the aid of (2.3), (2.4) results in

$$
\Phi\left(u_{0}\right)-\lambda \Psi\left(u_{0}\right) \leq \Phi(u)-\lambda \Psi(u), \quad \forall u \in \Phi^{-1}(]-\infty, r[) .
$$

Taking $u_{1}=u_{0}$ proves the other case and hence, $u_{1} \in \Phi^{-1}(]-\infty, r[)$ is a local minima of $J_{\lambda}$.

Now we prove the existence of a local minima $u_{2} \in \Phi^{-1}(] r, \infty[)$. Since $\lambda>\frac{1}{\varphi_{2}(r)}$, there exists $\bar{v} \in D(j)$ such that $\Phi(\bar{v})>r$ and

$$
\Phi(\bar{v})-\lambda \Psi(\bar{v})<r-\lambda \sup _{\Phi(x) \leq r} \Psi(x) .
$$

Let us introduce a functional $\Phi_{r}: X \rightarrow \mathbb{R}$ defined by

$$
\Phi_{r}(x):=\max \{\Phi(x), r\} .
$$

Clearly, $\Phi_{r}$ is sequentially weakly lower semicontinuous and coercive. Then, $J:=$ $\Phi_{r}-\lambda \Psi$ has the same properties, so there exists a global minimum $v_{0}$ of $J$, that is,

$$
\Phi_{r}\left(v_{0}\right)-\lambda \Psi\left(v_{0}\right) \leq \Phi_{r}(x)-\lambda \Psi(x), \quad \forall x \in X .
$$

Let us show that $v_{0} \in \Phi^{-1}(] r, \infty[)$. We argue indirectly and assume that $v_{0} \in$ $\left.\left.\Phi^{-1}(]-\infty, r\right]\right)$. Then, due to (2.6) with the special choice $x=\bar{v}$ and the fact that $\Phi(\bar{u})>r$, it follows

$$
r-\lambda \Psi\left(v_{0}\right) \leq \Phi(\bar{v})-\lambda \Psi(\bar{v}) .
$$

Applying (2.5) yields

$$
r-\lambda \Psi\left(v_{0}\right) \leq \Phi(\bar{v})-\lambda \Psi(\bar{v})<r-\lambda \Psi\left(v_{0}\right),
$$

which is a contradiction. Hence, $u_{2}:=v_{0} \in \Phi^{-1}(] r, \infty[)$ is a local minima of $J_{\lambda}$. Now, we may apply Corollary 2.1 in [35] to obtain the existence of at least three critical points of $J_{\lambda}$. This completes the proof.

Now, we present a variant of Theorem 2.1 where the (PS)-condition and the boundedness from below of $J_{\lambda}$ are not required. To this end, let $K$ be a non-empty closed convex subset of $X$ containing the zero of $X$ and let $j: X \rightarrow]-\infty,+\infty$ ] be defined as

$$
j(u)= \begin{cases}0, & \text { if } u \in K, \\ +\infty, & \text { otherwise. }\end{cases}
$$


Clearly, $j$ is convex, proper and lower semicontinuous. So, we can consider the functionals as defined in (2.2). Assume also that

1. $\inf _{X} \Phi=\Phi(0)=\Psi(0)=0$;

2. $\Phi$ is convex.

Moreover, for fixed $\lambda>0$, suppose that

3. for all $x_{1}, x_{2} \in X$ which are local minima of the functional $\Phi-\lambda \Psi$ such that $\Psi\left(x_{1}\right) \geq 0$ and $\Psi\left(x_{2}\right) \geq 0$, one has

$$
\inf _{t \in[0,1]} \Psi\left(t x_{1}+(1-t) x_{2}\right) \geq 0 ;
$$

4. there exist a real Banach space $\tilde{X}$ and a locally Lipschitz function $\tilde{\Theta}_{\lambda}$ : $\tilde{X} \rightarrow \mathbb{R}$ such that $X$ is compactly embedded in $\tilde{X}$ and $\left.\tilde{\Theta}_{\lambda}\right|_{X}=\Theta_{\lambda}$.

We obtain the following result.

Theorem 2.2. Assume that there are $r_{1}, r_{2}>0$ and $\bar{v} \in K$, with $2 r_{1}<\Phi(\bar{v})<$ $r_{2} / 2$, such that

$$
\begin{aligned}
& \frac{\sup _{u \in \Phi^{-1}(]-\infty, r_{1}[)} \Upsilon(u)}{r_{1}}<\frac{2}{3} \frac{\Upsilon(\bar{v})}{\Phi(\bar{v})} ; \\
& \frac{\sup _{u \in \Phi^{-1}(]-\infty, r_{2}[)} \Upsilon(u)}{r_{2}}<\frac{1}{3} \frac{\Upsilon(\bar{v})}{\Phi(\bar{v})} .
\end{aligned}
$$

Furthermore, suppose that $\Phi(u) \geq r_{2}$ for all $u \in \partial K$. Then, for each $\lambda \in \bar{\Lambda}$, where $\bar{\Lambda}$ is given through

$$
\bar{\Lambda}:=] \frac{3}{2} \frac{\Phi(\bar{v})}{\Upsilon(\bar{v})}, \min \left\{\frac{r_{1}}{\sup _{u \in \Phi^{-1}(]-\infty, r_{1}[)} \Upsilon(u)}, \frac{r_{2} / 2}{\sup _{u \in \Phi^{-1}(]-\infty, r_{2}[)} \Upsilon(u)}\right\}[,
$$

$J_{\lambda}$ has three distinct critical points $u_{i} \in K(i=1,2,3)$ such that $\Phi\left(u_{i}\right)<r_{2}$ for $i \in\{1,2,3\}$.

Proof. Put $\rho_{1}=r_{1}$ and $\rho_{2}=r_{2} / 2$. Because of $0 \in K$ and the definition of $j$, one has

$$
\varphi_{1}\left(\rho_{1}\right) \leq \frac{\sup _{u \in \Phi^{-1}(]-\infty, \rho_{1}[)} \Psi(u)}{\rho_{1}} \leq \frac{\sup _{u \in \Phi^{-1}(]-\infty, r_{1}[)} \Upsilon(u)}{r_{1}}
$$

and

$$
\varphi_{1}\left(\rho_{2}\right) \leq \frac{\sup _{u \in \Phi^{-1}(]-\infty, \rho_{2}[)} \Psi(u)}{\rho_{2}} \leq \frac{\sup _{u \in \Phi^{-1}(]-\infty, r_{2}[)} \Upsilon(u)}{r_{2} / 2} .
$$

On the other hand, since (2.7) implies $\sup _{u \in \Phi^{-1}(]-\infty, \rho_{1}[)} \Psi(u)<\Psi(\bar{v})$ and thanks to $\Phi(u) \geq 0$ for all $u \in X$, we obtain

$$
\inf _{u \in \Phi^{-1}(]-\infty, \rho_{1}[)} \sup _{v \in \Phi^{-1}\left(\left[\rho_{1}, \rho_{2}[)\right.\right.} \frac{\Psi(v)-\Psi(u)}{\Phi(v)-\Phi(u)} \geq \frac{2}{3} \frac{\Psi(\bar{v})}{\Phi(\bar{v})} .
$$

Hence,

$$
\max \left\{\varphi_{1}\left(\rho_{1}\right), \varphi_{1}\left(\rho_{2}\right)\right\}<\inf _{u \in \Phi^{-1}(]-\infty, \rho_{1}[)} \sup _{v \in \Phi^{-1}\left(\left[\rho_{1}, \rho_{2}[)\right.\right.} \frac{\Psi(v)-\Psi(u)}{\Phi(v)-\Phi(u)} .
$$

Therefore, owing to [3, Theorem 3.1] the functional $J_{\lambda}$ admits two local minima $u_{1}, u_{2} \in K$ such that $\Phi\left(u_{1}\right)<\rho_{1}$ and $\rho_{1} \leq \Phi\left(u_{2}\right)<\rho_{2}$.

Now put $\Upsilon_{r_{2} / 2 \lambda}(u)=\min \left\{\Upsilon(u), r_{2} / 2 \lambda\right\}$ and $\mathfrak{F}_{\lambda}(u)=\Phi(u)-\lambda \Upsilon_{r_{2} / 2 \lambda}(u)+\lambda j(u)$ for all $u \in X$. Since $\Phi-\lambda \Upsilon_{r_{2} / 2 \lambda}$ is coercive, then [35, Proposition 2.3] ensures that 
$\mathfrak{F}_{\lambda}$ satisfies the $(P S)_{c^{-}}$-condition for all $c \in \mathbb{R}$. Put $\bar{c}=\inf _{\gamma \in \Gamma} \max _{t \in[0,1]} \mathfrak{F}_{\lambda}(\gamma(t))$, where $\Gamma=\left\{\gamma \in C([0,1]): \gamma(0)=u_{1}, \gamma(1)=u_{2}\right\}$. It follows that

$$
\bar{c} \leq \max _{t \in[0,1]} \mathfrak{F}_{\lambda}\left(t u_{1}+(1-t) u_{2}\right) \leq \max _{t \in[0,1]}\left(t \Phi\left(u_{1}\right)+(1-t) \Phi\left(u_{2}\right)\right)<\rho_{2} .
$$

Since

$$
\mathfrak{F}_{\lambda}(u)=\Phi(u)-\lambda \Upsilon_{r_{2} / 2 \lambda}(u) \geq \Phi(u)-\lambda r_{2} / 2 \lambda \geq r_{2} / 2>\bar{c}, \quad \text { for all } u \in \partial K,
$$

we have that $\mathfrak{F}_{\lambda}^{\bar{c}}=\left\{x \in X: \mathfrak{F}_{\lambda}(x) \geq \bar{c}\right\}$ is closed (see [33, Lemma 2.1]). Therefore, [33, Theorem 4.2] ensures the existence of a critical point $u_{3}$ of $\mathfrak{F}_{\lambda}$ such that $\bar{c}=$ $\mathfrak{F}_{\lambda}\left(u_{3}\right)$. We claim that $\Upsilon\left(u_{3}\right)<r_{2} / 2 \lambda$. Arguing by contradiction, we assume that $\Upsilon\left(u_{3}\right) \geq r_{2} / 2 \lambda$. So, one has $\mathfrak{F}_{\lambda}\left(u_{3}\right)=\bar{c}<r_{2} / 2$, that is

$$
\Phi\left(u_{3}\right)-\lambda \Upsilon_{r_{2} / 2 \lambda}\left(u_{3}\right)<r_{2} / 2, \quad \Phi\left(u_{3}\right)-r_{2} / 2<r_{2} / 2, \quad \Phi\left(u_{3}\right)<r_{2} .
$$

Therefore, since $\lambda \in \bar{\Lambda}$ and, in particular,

$$
\lambda<\frac{r_{2} / 2}{\sup _{u \in \Phi^{-1}(]-\infty, r_{2}[)} \Upsilon(u)},
$$

we conclude that $\Upsilon\left(u_{3}\right)<r_{2} / 2 \lambda$. But this is a contradiction, so our claim is proved. It follows that $u_{3}$ is also a critical point of $J_{\lambda}$. This completes the proof.

In the following, we consider an equivalent norm on the space $W^{1, p}(\Omega)$ given by

$$
\|u\|_{W^{1, p}(\Omega)}:=\left(\int_{\Omega}\left(|\nabla u(x)|^{p}+q(x)|u(x)|^{p}\right) d x\right)^{\frac{1}{p}},
$$

(see for instance [38, Section 1.1.15]).

\section{THE CASE $p>N$}

In this section we prove the existence of multiple solutions to problem (1.1) if $p>N$. We recall that if $p>N$, the space $W^{1, p}(\Omega)$ is compactly embedded in $C(\bar{\Omega})$, that is, there exists a positive constant $c$ such that

$$
\|u\|_{C(\bar{\Omega})} \leq c\|u\|_{W^{1, p}(\Omega)} .
$$

First, we suppose there exist two constants $a_{1}, a_{2}>0$ such that

$$
\frac{\max _{|t| \leq a_{1}}(-F(t))}{a_{1}^{p}}<\frac{1}{c^{p}\|q\|_{L^{1}(\Omega)}} \frac{\left(-F\left(a_{2}\right)\right)}{a_{2}^{p}}
$$

and take $\lambda \in \Lambda$, where $\Lambda$ is given by

$$
\Lambda:=] \frac{\|q\|_{L^{1}(\Omega)}}{p\|\alpha\|_{L^{1}(\Omega)}} \frac{a_{2}^{p}}{\left(-F\left(a_{2}\right)\right)}, \frac{1}{p c^{p}\|\alpha\|_{L^{1}(\Omega)}} \frac{a_{1}^{p}}{\max _{|t| \leq a_{1}}(-F(t))}[.
$$

Thanks to (3.1) we observe that the interval $\Lambda$ is non-empty. 
Put

$$
\begin{aligned}
& \delta:=\min \left\{\frac{a_{1}^{p}-p c^{p} \lambda\|\alpha\|_{L^{1}(\Omega)} \max _{|t| \leq a_{1}}(-F(t))}{p c^{p}\|\beta\|_{L^{1}(\Gamma)} \max _{|t| \leq a_{1}}(-G(t))},\right. \\
& \left.\frac{p \lambda\|\alpha\|_{L^{1}(\Omega)}\left(-F\left(a_{2}\right)\right)-a_{2}^{p}\|q\|_{L^{1}(\Omega)}}{p\|\beta\|_{L^{1}(\Gamma)} \min \left\{0,-G\left(a_{2}\right)\right\}}\right\}, \\
& \tilde{\delta}=\frac{1}{\max \left\{0, p \max \left\{\|\alpha\|_{L^{1}(\Omega)},\|\beta\|_{L^{1}(\Gamma)}\right\} c^{p} \limsup _{|\xi| \rightarrow+\infty} \frac{(-G(\xi))}{|\xi|^{p}}\right\}} \\
& \bar{\delta}:=\min \{\delta, \tilde{\delta}\} .
\end{aligned}
$$

Since $\lambda \in \Lambda$, a simple computation shows that $\delta>0$.

If $\lim \sup _{|\xi| \rightarrow+\infty} \frac{(-G(\xi))}{|\xi|^{p}} \leq 0$ and $\max _{|t| \leq a_{1}}(-G(t))=0$ as well as $G\left(a_{2}\right) \geq 0$ we read $\bar{\delta}=+\infty$.

Our main result in this section is the following.

Theorem 3.1. Let $\alpha \in L^{1}(\Omega), \beta \in L^{1}(\Gamma)$ be two non-negative and non-zero functions. Let $f: \mathbb{R} \rightarrow \mathbb{R}$ be a locally essentially bounded function and put $F(\xi)=\int_{0}^{\xi} f(t) d t$ for every $\xi \in \mathbb{R}$. Assume that

(H1) $\frac{\max _{|t| \leq a_{1}}(-F(t))}{a_{1}^{p}}<\frac{1}{c^{p}\|q\|_{L^{1}(\Omega)}} \frac{\left(-F\left(a_{2}\right)\right)}{a_{2}^{p}}$;

(H2) $\limsup _{|\xi| \rightarrow+\infty} \frac{(-F(\xi))}{\xi^{p}} \leq 0$,

with positive constants $a_{1}, a_{2}$ satisfying $a_{1}<a_{2}$ and $a_{2} \in K$. Then, for each $\lambda \in \Lambda$, where $\Lambda$ is defined in (3.2), and for each locally essentially bounded function $g: \mathbb{R} \rightarrow \mathbb{R}$, whose potential $G(\xi)=\int_{0}^{\xi} g(t) d t$ for every $\xi \in \mathbb{R}$ satisfies

(H3) $\limsup _{|\xi| \rightarrow+\infty} \frac{(-G(\xi))}{|\xi|^{p}}<+\infty$,

there exists $\bar{\delta}>0$ given by (3.3) such that, for each $\mu \in[0, \bar{\delta}[$, problem (1.1) has at least three distinct solutions.

Proof. Our aim is to apply Theorem 2.1. Let $\lambda, g$ and $\mu$ be fixed satisfying the assumptions and let $X$ be the space $W^{1, p}(\Omega)$ equipped with the norm $\|\cdot\|_{W^{1, p}(\Omega)}$. We set, for any $u \in X$,

$$
\begin{aligned}
& \Phi(u):=\frac{1}{p}\|u\|_{W^{1, p}(\Omega)}^{p}, \quad j(u)= \begin{cases}0, & \text { if } u \in K, \\
+\infty, & \text { otherwise },\end{cases} \\
& \Upsilon(u)=\int_{\Omega} \alpha(x)[-F(u(x))] d x+\frac{\mu}{\lambda} \int_{\Gamma} \beta(x)[-G(\gamma u(x)] d \sigma, \\
& \Psi(u):=\Upsilon(u)-j(u), \quad J_{\lambda}(u):=\Phi(u)-\lambda \Psi(u) .
\end{aligned}
$$


Then

$$
\begin{aligned}
& J_{\lambda}(u) \\
& =\frac{1}{p}\|u\|_{W^{1, p}(\Omega)}^{p}-\lambda \int_{\Omega} \alpha(x)[-F(u(x))] d x-\mu \int_{\Gamma} \beta(x)[-G(\gamma u(x)] d \sigma+\lambda j(u) .
\end{aligned}
$$

Let $r=\frac{1}{p}\left(\frac{a_{1}}{c}\right)^{p}$, then $\Phi\left(a_{2}\right)>r$, that means $\left.r \in\right] \inf _{X} \Phi, \sup _{X} \Phi[=] 0, \sup _{X} \Phi[$. Let $v \in \Phi^{-1}(]-\infty, r[)$. Then due to $\frac{1}{p}\|v\|_{W^{1, p}(\Omega)}^{p}<r$ combined with $\|v\|_{C(\bar{\Omega})} \leq$ $c\|v\|_{W^{1, p}(\Omega)}$, we have $|v(x)| \leq a_{1}$ for every $x \in \Omega$. We obtain

$$
\begin{aligned}
& \varphi_{1}(r) \\
& \leq \frac{\sup _{\|w\|^{p} \leq p r}\left(\int_{\Omega} \alpha(x)[-F(w(x))] d x+\frac{\mu}{\lambda} \int_{\Gamma} \beta(x)[-G(\gamma w(x))] d \sigma-j(w)\right)}{r} \\
& \leq \frac{\|\alpha\|_{L^{1}(\Omega)} \max _{|t| \leq a_{1}}(-F(t))+\frac{\mu}{\lambda}\|\beta\|_{L^{1}(\Gamma)} \max _{|t| \leq r}(-G(r))}{r} \\
& =p c^{p}\|\alpha\|_{L^{1}(\Omega)} \frac{\max _{|t| \leq a_{1}}(-F(t))}{a_{1}^{p}}+p c^{p} \frac{\mu}{\lambda}\|\beta\|_{L^{1}(\Gamma)} \frac{\max _{|t| \leq a_{1}}(-G(t))}{a_{1}^{p}} .
\end{aligned}
$$

On the other side we have

$$
\frac{\Psi\left(a_{2}\right)}{\Phi\left(a_{2}\right)} \geq \frac{p\|\alpha\|_{L^{1}(\Omega)}}{\|q\|_{L^{1}(\Omega)}} \frac{\left[-F\left(a_{2}\right)\right]}{a_{2}^{p}}+\frac{\|\beta\|_{L^{1}(\Gamma)} \mu p}{\lambda\|q\|_{L^{1}(\Omega)}} \frac{\min \left\{0,-G\left(a_{2}\right)\right\}}{a_{2}^{p}} .
$$

As $\mu<\delta$ (see (3.3)), it follows

$$
\begin{aligned}
& \mu<\frac{a_{1}^{p}-p c^{p} \lambda\|\alpha\|_{L^{1}(\Omega)} \max _{|t| \leq a_{1}}(-F(t))}{p c^{p}\|\beta\|_{L^{1}(\Gamma)} \max _{|t| \leq a_{1}}(-G(t))} \quad \text { and } \\
& \mu<\frac{p \lambda\|\alpha\|_{L^{1}(\Omega)}\left(-F\left(a_{2}\right)\right)-a_{2}^{p}\|q\|_{L^{1}(\Omega)}}{p\|\beta\|_{L^{1}(\Gamma)} \min \left\{0,-G\left(a_{2}\right)\right\}}
\end{aligned}
$$

From (3.4) we obtain

$$
\frac{p c^{p}\|\alpha\|_{L^{1}(\Omega)} \max _{|t| \leq a_{1}}(-F(t))}{a_{1}^{p}}+\frac{\mu}{\lambda} \frac{p c^{p}\|\beta\|_{L^{1}(\Gamma)} \max _{|t| \leq a_{1}}(-G(t))}{a_{1}^{p}}<\frac{1}{\lambda},
$$

respectively,

$$
\frac{p\|\alpha\|_{L^{1}(\Omega)}}{\|q\|_{L^{1}(\Omega)}} \frac{\left[-F\left(a_{1}\right)\right]}{a_{2}^{p}}+\frac{\|\beta\|_{L^{1}(\Gamma)} \mu p}{\lambda\|q\|_{L^{1}(\Omega)}} \frac{\min \left\{0,-G\left(a_{2}\right)\right\}}{a_{2}^{p}}>\frac{1}{\lambda} .
$$

Hence

$$
\varphi_{1}(r)<\frac{1}{\lambda}<\frac{\Psi\left(a_{2}\right)}{\Phi\left(a_{2}\right)} .
$$

In particular, we obtain from the calculations above that

$$
\frac{\sup _{\Phi(x)<r} \Psi(x)}{r} \leq \frac{\sup _{\Phi(x) \leq r} \Psi(x)}{r}<\frac{\Psi\left(a_{2}\right)}{\Phi\left(a_{2}\right)},
$$

where $0<r<\Phi\left(a_{2}\right)$. This leads to

$$
\varphi_{2}(r) \geq \frac{\Psi\left(a_{2}\right)-\sup _{\Phi(x) \leq r} \Psi(x)}{\Phi\left(a_{2}\right)-r} \geq \frac{\Psi\left(a_{2}\right)-r \frac{\Psi\left(a_{2}\right)}{\Phi\left(a_{2}\right)}}{\Phi\left(a_{2}\right)-r}=\frac{\Psi\left(a_{2}\right)}{\Phi\left(a_{2}\right)} .
$$

Combining (3.5) and (3.6) guarantees

$$
\varphi_{1}(r)<\frac{1}{\lambda}<\varphi_{2}(r) .
$$


Let us now prove that $J_{\lambda}$ is coercive. Since $\mu<\bar{\delta}$ and due to (H3), there exists a constant $k>0$ such that

$$
\limsup _{|\eta| \rightarrow+\infty} \frac{\max _{|t| \leq \eta}(-G(t))}{\eta^{p}}<k \quad \text { and } \quad \mu k<\frac{1}{\max \left\{\|\alpha\|_{L^{1}(\Omega)},\|\beta\|_{L^{1}(\Gamma)}\right\} p c^{p}} .
$$

Thus, we get an estimate of the form

$$
-G(s) \leq k s^{p}+b_{1} \quad \forall s \in \mathbb{R},
$$

with a non-negative constant $b_{1}$. Putting $\tau$ fixed such that

$$
0<\tau<\left(\frac{1}{\max \left\{\|\alpha\|_{L^{1}(\Omega)},\|\beta\|_{L^{1}(\Gamma)}\right\} p c^{p}}-\mu k\right) \frac{1}{\lambda}
$$

yields, with the aid of hypothesis (H2),

$$
-F(s) \leq \tau s^{p}+b_{2}, \quad \forall s \in \mathbb{R},
$$

with $b_{2}$ being non-negative. From (3.7) and (3.8) applied on the functional $J_{\lambda}$ it follows, for $u \in D(j)$ (otherwise we are done),

$$
\begin{aligned}
& J_{\lambda}(u)=\Phi(u)-\lambda \Psi(u) \\
& \geq\left[\frac{1}{p}-\lambda \tau c^{p} \max \left\{\|\alpha\|_{L^{1}(\Omega)},\|\beta\|_{L^{1}(\Gamma)}\right\}-\mu k c^{p} \max \left\{\|\alpha\|_{L^{1}(\Omega)},\|\beta\|_{L^{1}(\Gamma)}\right\}\right] \\
& \quad \times\|u\|_{W^{1, p}(\Omega)}^{p}-\lambda b_{2}\|\alpha\|_{L^{1}(\Omega)}-\mu b_{1}\|\beta\|_{L^{1}(\Gamma)},
\end{aligned}
$$

where

$$
\frac{1}{p}-\lambda \tau c^{p} \max \left\{\|\alpha\|_{L^{1}(\Omega)},\|\beta\|_{L^{1}(\Gamma)}\right\}-\mu k c^{p} \max \left\{\|\alpha\|_{L^{1}(\Omega)},\|\beta\|_{L^{1}(\Gamma)}\right\}>0 .
$$

This proves the coercivity of $J_{\lambda}$. In order to prove the Palais-Smale condition for $J_{\lambda}$, we have to apply [35, Proposition 2.3]. Now we are able to apply Theorem 2.1 obtaining the existence of three distinct critical points of $J_{\lambda}$ denoted by $u_{1}, u_{2}$ and $u_{3}$. Let $u:=u_{1}$ be the first critical point of $J_{\lambda}$, then one has

$$
(\Phi-\lambda \Upsilon)^{\circ}(u ; v-u)+\lambda j(v)-\lambda j(u) \geq 0 \quad \text { for all } v \in X .
$$

Clearly, from (3.9) we see at once that $u \in K$ (otherwise (3.9) fails). Hence it follows

$$
(\Phi-\lambda \Upsilon)^{\circ}(u ; v-u) \geq 0 \quad \text { for all } v \in K .
$$

The left-hand side of (3.10) can be estimated using (2.1)

$$
\Phi^{\prime}(u ; v-u)+\lambda(-\Upsilon)^{\circ}(u ; v-u) \geq 0 \quad \text { for all } v \in K,
$$

which means

$$
\begin{aligned}
& \int_{\Omega}|\nabla u(x)|^{p-2} \nabla u(x) \cdot \nabla(v(x)-u(x)) d x \\
& \quad+\int_{\Omega} q(x)|u(x)|^{p-2} u(x)(v(x)-u(x)) d x+\lambda(-\Upsilon)^{\circ}(u, v-u) \geq 0,
\end{aligned}
$$


for all $v \in K$. Applying again (2.1) combined with the formula (2) in [18, p. 77] leads to

$$
\begin{aligned}
\lambda(-\Upsilon)^{\circ}(u ; v-u) \leq & \lambda \int_{\Omega} \alpha(x) F^{\circ}(u(x) ; v(x)-u(x)) d x \\
& +\mu \int_{\Gamma} \beta(x) G^{\circ}(\gamma u(x) ; \gamma v(x)-\gamma u(x)) d \sigma .
\end{aligned}
$$

Finally, from (3.11) and (3.12) we have

$$
\begin{aligned}
& \int_{\Omega}|\nabla u(x)|^{p-2} \nabla u(x) \cdot \nabla(v(x)-u(x)) d x \\
& \quad+\int_{\Omega} q(x)|u(x)|^{p-2} u(x)(v(x)-u(x)) d x \\
& \quad+\lambda \int_{\Omega} \alpha(x) F^{\circ}(u(x) ; v(x)-u(x)) d x \\
& \quad+\mu \int_{\partial \Omega} \beta(x) G^{\circ}(\gamma u(x) ; \gamma v(x)-\gamma u(x)) d \sigma \geq 0 \text { for all } v \in K .
\end{aligned}
$$

This proves that $u=u_{1}$ is a solution of our problem (1.1). The same calculations can be done for $u_{2}$, respectively $u_{3}$, which completes the proof of the theorem.

Let us give a simple example to Theorem 3.1.

Example 3.2. Let $N<p \leq 11$, let $K$ be a closed convex subset of $W^{1, p}(\Omega)$ with $2 \in K$ and let $q(x) \equiv \frac{1}{c^{p}|\Omega|}$ for all $x \in \Omega$. We define the function $f: \mathbb{R} \rightarrow \mathbb{R}$ by

$$
f(t)= \begin{cases}2 e^{-t^{2}} t & \text { if } t<-1 \text { and } t>2, \\ -2 e^{-t^{2}} t^{17}\left(9-t^{2}\right)-1, & \text { if }-1 \leq t \leq 2\end{cases}
$$

Putting $a_{1}=1$ and $a_{2}=2$ we conclude

$$
\frac{\max _{|t| \leq a_{1}}[-F(t)]}{a_{1}^{p}}=\max _{|t| \leq 1}\left[e^{-t^{2}} t^{18}+t\right]=e^{-1}+1<\frac{e^{-2^{2}} 2^{18}+2}{2^{p}}=\frac{\left[-F\left(a_{2}\right)\right]}{a_{2}^{p}} .
$$

Hence, condition (H1) of Theorem 3.1 is satisfied and (H2) is obviously true. Let $g: \mathbb{R} \rightarrow \mathbb{R}$ be defined by

$$
g(t)= \begin{cases}-t^{q} & \text { if } t \leq 0 \\ -2 t^{q} & \text { if } t>0\end{cases}
$$

with $q<p-1$. Then, assumption (H3) is also fulfilled. The application of Theorem 3.1 yields the existence of three nontrivial solutions to (1.1).

Let us now show a special version of Theorem 3.1 when $K$ is a ball. First, we show that every solution of (1.1) is non-negative provided the functions $f$ and $g$ are non-positive along with the assumption that the closed convex set $K$ has partially lattice structure.

Proposition 3.3. Let $p>N$ and assume that $f(t) \leq 0$ and $g(t) \leq 0$ for almost all $t \in \mathbb{R}$. Let $u \in K$ be a solution of (1.1) such that $u^{+}=\max \{u, 0\} \in K$. Then, $u(x) \geq 0$ for all $x \in \Omega$. 
G. BONANNO AND P. WINKERT

Proof. Let $u \in K \subset W^{1, p}(\Omega)$ be a solution of (1.1). Since $f$ and $g$ are non-positive, it is clear that $F$ and $G$ are non-increasing. Hence, we have $F^{\circ}(\xi ; \eta-\xi) \leq 0$ and $G^{\circ}(\xi ; \eta-\xi) \leq 0$ for all $\xi, \eta \in \mathbb{R}$ satisfying $\eta-\xi \geq 0$. Therefore, for all $v \in K$ such that $v(x)-u(x) \geq 0$ for all $x \in \Omega$, it follows

$$
\begin{aligned}
& \int_{\Omega} \lambda \alpha(x) F^{\circ}(u(x) ; v(x)-u(x)) d x \leq 0, \\
& \int_{\Gamma} \mu \beta(x) G^{\circ}(\gamma u(x) ; \gamma v(x)-\gamma u(x)) d \sigma \leq 0 .
\end{aligned}
$$

Applying this to (1.1) yields

$$
\begin{aligned}
& \int_{\Omega}|\nabla u(x)|^{p-2} \nabla u(x) \cdot \nabla(v(x)-u(x)) d x \\
& \quad+\int_{\Omega} q(x)|u(x)|^{p-2} u(x)(v(x)-u(x)) d x \geq 0,
\end{aligned}
$$

for all $v \in K$ such that $v(x)-u(x) \geq 0$ for all $x \in \Omega$.

Now, let $A:=\{x \in \Omega: u(x)<0\}$. Since $u^{+}(x) \geq u(x)$ for all $x \in \Omega$ and thanks to $u^{+} \in K$, we may choose $v=u^{+} \in K$ as test function to derive

$$
-\int_{A}|\nabla(u(x))|^{p} d x-\int_{A} q(x)|u(x)|^{p} d x \geq 0,
$$

meaning

$$
\|u\|_{W^{1, p}(A)} \leq 0
$$

This proves the non-negativity of $u$.

Remark 3.4. Note that if $K$ is the ball $B(0, M)$ with center 0 and radius $M>0$ it clearly holds $u^{+} \in K$ which can be easily seen from the estimate $\left|u^{+}(x)\right| \leq|u(x)|$ for all $x \in \Omega$.

Next, suppose the existence of three positive constants $a_{1}, a_{2}$, and $a_{3}$ such that

$$
\frac{\left[-F\left(a_{1}\right)\right]}{a_{1}^{p}}<\frac{2}{3 c^{p}\|q\|_{L^{1}(\Omega)}} \frac{\left[-F\left(a_{2}\right)\right]}{a_{2}^{p}}, \quad \frac{\left[-F\left(a_{3}\right)\right]}{a_{3}^{p}}<\frac{1}{3 c^{p}\|q\|_{L^{1}(\Omega)}} \frac{\left[-F\left(a_{2}\right)\right]}{a_{2}^{p}} .
$$

Taking

$$
\begin{aligned}
\Lambda= & \frac{3\|q\|_{L^{1}(\Omega)}}{2 p\|\alpha\|_{L^{1}(\Omega)}} \frac{a_{2}^{p}}{\left[-F\left(a_{2}\right)\right]}, \\
& \min \left\{\frac{1}{p c^{p}\|\alpha\|_{L^{1}(\Omega)}} \frac{a_{1}^{p}}{\left[-F\left(a_{1}\right)\right]}, \frac{1}{p c^{p}\|\alpha\|_{L^{1}(\Omega)}} \frac{a_{3}^{p}}{2\left[-F\left(a_{3}\right)\right]}\right\}[
\end{aligned}
$$

we see that $\Lambda$ is non-empty. Let $\lambda \in \Lambda$ and put the number

$$
\begin{aligned}
\delta=\min \left\{\frac{a_{1}^{p}-\lambda p c^{p}\|\alpha\|_{L^{1}(\Omega)}\left[-F\left(a_{1}\right)\right]}{p c^{p}\|\beta\|_{L^{1}(\Gamma)}\left[-G\left(a_{1}\right)\right]},\right. \\
\\
\left.\frac{a_{3}^{p}-\lambda 2 p c^{p}\|\alpha\|_{L^{1}(\Omega)}\left[-F\left(a_{3}\right)\right]}{2 p c^{p}\|\beta\|_{L^{1}(\Gamma)}\left[-G\left(a_{3}\right)\right]}\right\},
\end{aligned}
$$

which is obviously positive. If $K$ is the ball $B(0, M)$, we have the following result. 
Theorem 3.5. Let $K=B(0, M)$ and let $\alpha \in L^{1}(\Omega), \beta \in L^{1}(\Gamma)$ be two non-negative and non-zero functions. Let $f: \mathbb{R} \rightarrow \mathbb{R}$ be a locally essentially bounded function such that $f(t) \leq 0$ for a.a. $t \in \mathbb{R}$. Put $F(\xi)=\int_{0}^{\xi} f(t) d t$ for every $\xi \in \mathbb{R}$ and suppose that

$$
\begin{aligned}
& \frac{\left[-F\left(a_{1}\right)\right]}{a_{1}^{p}}<\frac{2}{3 c^{p}\|q\|_{L^{1}(\Omega)}} \frac{\left[-F\left(a_{2}\right)\right]}{a_{2}^{p}} \\
& \frac{\left[-F\left(a_{3}\right)\right]}{a_{3}^{p}}<\frac{1}{3 c^{p}\|q\|_{L^{1}(\Omega)}} \frac{\left[-F\left(a_{2}\right)\right]}{a_{2}^{p}}
\end{aligned}
$$

with positive constants $a_{1}, a_{2}$ and $a_{3}$ satisfying

$$
2^{\frac{1}{p}} a_{1}<c\|q\|_{L^{1}(\Omega)}^{\frac{1}{p}} a_{2}<\frac{1}{2^{\frac{1}{p}}} a_{3},
$$

with $a_{2} \in K$ and $a_{3} \leq c\|q\|_{L^{1}(\Omega)}^{\frac{1}{p}} M$.

Then, for each $\lambda \in \Lambda$, where $\Lambda$ is defined in (3.13), and for each locally essentially bounded function $g: \mathbb{R} \rightarrow \mathbb{R}$ such that $g(t) \leq 0$ for a.a. $x \in \mathbb{R}$ and with $G(\xi):=$ $\int_{0}^{\xi} g(t) d t$ for every $\xi \in \mathbb{R}$, there exists $\delta>0$ given by (3.14) such that for each $\mu \in\left[0, \delta\left[\right.\right.$, problem (1.1) has at least three distinct solutions $u_{i}(i=1,2,3)$ satisfying $0 \leq u_{i}(x)<a_{3}$ for all $x \in \Omega$ and all $i \in\{1,2,3\}$.

Proof. The ideas of the proof are mainly based on the proof of Theorem 3.1 with the difference that we want to apply Theorem 2.2 instead of Theorem 2.1. Let $\Phi, j, \Upsilon, \Psi$ and $J_{\lambda}$ as in the proof of Theorem 3.1 and let $r_{1}=\frac{1}{p}\left(\frac{a_{1}}{c}\right)^{p}$. Then we conclude

$$
\frac{\sup _{u \in \Phi^{-1}(]-\infty, r_{1}[)} \Upsilon(u)}{r_{1}} \leq p c^{p}\|\alpha\|_{L^{1}(\Omega)} \frac{\left[-F\left(a_{1}\right)\right]}{a_{1}^{p}}+p c^{p} \frac{\mu}{\lambda}\|\beta\|_{L^{1}(\Gamma)} \frac{\left[-G\left(a_{1}\right)\right]}{a_{1}^{p}} .
$$

On the other side, for $r_{2}=\frac{1}{p}\left(\frac{a_{3}}{c}\right)^{p}$ we obtain

$$
\frac{\sup _{u \in \Phi^{-1}(]-\infty, r_{2}[)} \Upsilon(u)}{r_{2}} \leq p c^{p}\|\alpha\|_{L^{1}(\Omega)} \frac{\left[-F\left(a_{3}\right)\right]}{a_{3}^{p}}+p c^{p} \frac{\mu}{\lambda}\|\beta\|_{L^{1}(\Gamma)} \frac{\left[-G\left(a_{3}\right)\right]}{a_{3}^{p}} .
$$

By assumption we have $\mu<\delta$ which results in

$$
\mu<\frac{a_{1}^{p}-\lambda p c^{p}\|\alpha\|_{L^{1}(\Omega)}\left[-F\left(a_{1}\right)\right]}{p c^{p}\|\beta\|_{L^{1}(\Gamma)}\left[-G\left(a_{1}\right)\right]} \quad \text { and } \quad \mu<\frac{a_{3}^{p}-\lambda 2 p c^{p}\|\alpha\|_{L^{1}(\Omega)}\left[-F\left(a_{3}\right)\right]}{2 p c^{p}\|\beta\|_{L^{1}(\Gamma)}\left[-G\left(a_{3}\right)\right]} \text {. }
$$

Combining these estimates above yields

$$
\frac{\sup _{u \in \Phi^{-1}(]-\infty, r_{1}[)} \Upsilon(u)}{r_{1}}<\frac{1}{\lambda} \quad \text { and } \quad \frac{\sup _{u \in \Phi^{-1}(]-\infty, r_{2}[)} \Upsilon(u)}{\frac{r_{2}}{2}}<\frac{1}{\lambda} .
$$

We observe that

$$
\frac{\Upsilon\left(a_{2}\right)}{\Phi\left(a_{2}\right)} \geq \frac{p\|\alpha\|_{L^{1}(\Omega)}}{\|q\|_{L^{1}(\Omega)}} \frac{\left[-F\left(a_{2}\right)\right]}{a_{2}^{p}} .
$$

Furthermore, since $\lambda \in \Lambda$, we have

$$
\frac{3\|q\|_{L^{1}(\Omega)}}{2 p\|\alpha\|_{L^{1}(\Omega)}} \frac{a_{2}^{p}}{\left[-F\left(a_{2}\right)\right]}<\lambda .
$$

Hence, it follows

$$
\frac{2}{3} \frac{\Upsilon\left(a_{2}\right)}{\Phi\left(a_{2}\right)}>\frac{1}{\lambda}
$$


Now, we see that from (3.16) and (3.17) the estimates in (2.7) and (2.8), respectively, are satisfied. Taking into account (3.15) a simple calculation leads to

$$
2 r_{1}<\Phi\left(a_{2}\right)<\frac{r_{2}}{2}
$$

Let $u \in \partial K$, then, due to $a_{3} \leq c\|q\|_{L^{1}(\Omega)}^{\frac{1}{p}} M$, we have

$$
\Phi(u)=\frac{1}{p} M^{p}\|q\|_{L^{1}(\Omega)} \geq \frac{1}{p}\left(\frac{a_{3}}{c}\right)^{p}=r_{2} .
$$

Now we may apply Theorem 2.2 obtaining the existence of three distinct critical points $u_{i} \in K(i=1,2,3)$ of $J_{\lambda}$ satisfying $\Phi\left(u_{i}\right)<r_{2}$ for all $x \in \Omega$ and $i=1,2,3$. Similar to the proof of Theorem 3.1 it can be easily shown that these critical points are solutions of our original problem (1.1). Thanks to Proposition 3.3 and Remark 3.4 we conclude that these solutions are non-negative and owing to the embedding $W^{1, p}(\Omega) \hookrightarrow C(\bar{\Omega})$, we see that $u_{i}(x)<a_{3}$ is satisfied for all $x \in \Omega$ and for $i=1,2,3$. This completes the proof of the theorem.

We close this section with an application of Theorem 3.5.

Example 3.6. Let $p>N$ with $p \geq 2$ and denote by $K=B(0, M)$ the ball with center zero and radius $M \geq 4$ while $q(x) \equiv \frac{1}{c^{p}|\Omega|}$ for all $x \in \Omega$. Further, let $g: \mathbb{R} \rightarrow \mathbb{R}$ be a non-positive, locally essentially bounded function and let $f: \mathbb{R} \rightarrow \mathbb{R}$ be defined through

$$
f(t)= \begin{cases}-h(t) & \text { if } t<0 \text { and } t \geq 4 \\ -1 & \text { if } 0 \leq t<1 \text { and } 2 \leq t<4, \\ -3 \cdot 2^{2 p} t^{2} & \text { if } 1 \leq t<2\end{cases}
$$

with a non-negative, locally essentially bounded function $h: \mathbb{R} \rightarrow \mathbb{R}$. Put

$$
a_{1}=1, \quad a_{2}=2, \quad a_{3}=4,
$$

then we have

$$
2^{\frac{1}{p}} a_{1}<c\|q\|_{L^{1}(\Omega)}^{\frac{1}{p}} a_{2}<\frac{1}{2^{\frac{1}{p}}} a_{3}, \quad a_{3} \leq c\|q\|_{L^{1}(\Omega)}^{\frac{1}{p}} M \quad \text { and } \quad a_{2} \in K .
$$

A simple calculation shows

$$
\begin{aligned}
\frac{\left[-F\left(a_{1}\right)\right]}{a_{1}^{p}} & =\frac{-\int_{0}^{a_{1}}-1 d t}{a_{1}^{p}}=a_{1}=1, \\
\frac{\left[-F\left(a_{2}\right)\right]}{a_{2}^{p}} & =\frac{\int_{0}^{a_{1}} 1 d t+\int_{a_{1}}^{a_{2}} 3 \cdot 2^{2 p} t^{2} d t}{a_{2}^{p}}=\frac{1+2^{2 p}\left(2^{3}-1\right)}{2^{p}}=7 \cdot 2^{p}+2^{-p}, \\
\frac{\left[-F\left(a_{3}\right)\right]}{a_{3}^{p}} & =\frac{\int_{0}^{a_{1}} 1 d t+\int_{a_{1}}^{a_{2}} 3 \cdot 2^{2 p} t^{2} d t+\int_{a_{2}}^{a_{3}} 1 d t}{a_{3}^{p}} \\
& =\frac{1+2^{2 p}\left(2^{3}-1\right)+(4-2)}{4^{p}}=2^{-2 p}+9 .
\end{aligned}
$$

Hence, due to $p \geq 2$, it results in

$$
\begin{aligned}
& \frac{\left[-F\left(a_{1}\right)\right]}{a_{1}^{p}}=1<\frac{14}{3} 2^{p}+\frac{2}{3} 2^{-p}=\frac{2}{3} \frac{\left[-F\left(a_{2}\right)\right]}{a_{2}^{p}} \\
& \frac{\left[-F\left(a_{3}\right)\right]}{a_{3}^{p}}=2^{-2 p}+9<\frac{7}{3} \cdot 2^{p}+\frac{1}{3} 2^{-p}=\frac{1}{3} \frac{\left[-F\left(a_{2}\right)\right]}{a_{2}^{p}} .
\end{aligned}
$$


Now we may apply Theorem 3.5 to obtain the existence of three distinct solutions $u_{i}$ $(i=1,2,3)$ to problem (1.1) which are bounded through $0 \leq u_{i}(x)<4$ for $i=1,2,3$.

\section{The CASE $p \leq N$}

In this section we study the case $p \leq N$. From now on it is supposed that $\alpha \in L^{\infty}(\Omega), \beta \in L^{\infty}(\Gamma)$ and there exist constants $b_{1}, b_{2} \geq 0$ such that

$$
|f(t)| \leq b_{1}+b_{2}|t|^{s-1}
$$

for all $t \in \mathbb{R}$ while $s \in] 1, \frac{N p}{N-p}[$ if $p<N$ as well as $s \in] 1,+\infty[$ if $p=N$. Thanks to the Sobolev embedding and the trace embedding there are positive constants $c_{s}$ and $C$ satisfying

$$
\begin{aligned}
\|u\|_{L^{s}(\Omega)} & \leq c_{s}\|u\|_{W^{1, p}(\Omega)}, \\
\|u\|_{L^{p}(\Gamma)} & \leq C\|u\|_{W^{1, p}(\Omega)}
\end{aligned}
$$

for all $u \in W^{1, p}(\Omega)$. Applying the constants $b_{1}, b_{2}$ from (4.1) along with the constants $c_{1}$ and $c_{s}$ in (4.2) we put

$$
K_{1}:=\|\alpha\|_{L^{\infty}(\Omega)} b_{1} c_{1} p^{\frac{1}{p}}, \quad K_{2}:=\|\alpha\|_{L^{\infty}(\Omega)} b_{2} c_{s}^{s} \frac{p^{\frac{s}{p}}}{s} .
$$

Now we assume there are two constants $a_{1}, a_{2}>0$ fulfilling $a_{1}<\left(\frac{\|q\|_{L^{1}(\Omega)}}{p}\right)^{\frac{1}{p}} a_{2}$ with $a_{2} \in K$ such that

$$
K_{1} \frac{1}{a_{1}^{p-1}}+K_{2} a_{1}^{s-p}<\frac{p\|\alpha\|_{L^{1}(\Omega)}}{\|q\|_{L^{1}(\Omega)}} \frac{\left[-F\left(a_{2}\right)\right]}{a_{2}^{p}} .
$$

Due to (4.4) we see at once that

$$
\widetilde{\Lambda}:=] \frac{\|q\|_{L^{1}(\Omega)}}{p\|\alpha\|_{L^{1}(\Omega)}} \frac{a_{2}^{p}}{\left[-F\left(a_{2}\right)\right]}, \frac{1}{K_{1} \frac{1}{a_{1}^{p-1}}+K_{2} a_{1}^{s-p}}[
$$

is non-empty.

Furthermore, let $g: \mathbb{R} \rightarrow \mathbb{R}$ be a locally essentially bounded function and set $G(\xi)=\int_{0}^{\xi} g(t) d t$ for $\xi \in \mathbb{R}$. We suppose that

$$
[-G(\xi)] \leq b_{3}|\xi|^{p}
$$

for all $\xi \in \mathbb{R}$ with $b_{3}$ being a non-negative constant. Similar to the case $p>N$ we put

$$
\begin{aligned}
& \delta:=\min \left\{\frac{1-\lambda\left(K_{1} \frac{1}{a_{1}^{p-1}}+K_{2} a_{1}^{s-p}\right)}{\|\beta\|_{L^{\infty}(\Gamma)} b_{3} C^{p} p}, \frac{1-\lambda\left(\frac{p\|\alpha\|_{L^{1}(\Omega)}}{\|q\|_{L^{1}(\Omega)}} \frac{\left[-F\left(a_{2}\right)\right]}{a_{2}^{p}}\right)}{\frac{p\|\beta\|_{L^{1}(\Gamma)}}{\|q\|_{L^{1}}(\Omega)} \frac{\min \left\{0,-G\left(a_{2}\right)\right\}}{a_{2}^{p}}}\right\}, \\
& \tilde{\delta}=\frac{1}{\max \left\{\|\alpha\|_{L^{\infty}(\Omega)},\|\beta\|_{L^{\infty}(\Gamma)}\right\} p c_{p}^{p} b_{3}}, \\
& \bar{\delta}:=\min \{\delta, \tilde{\delta}\},
\end{aligned}
$$

with $\lambda \in \widetilde{\Lambda}$.

Now we can formulate the main result in this section. 
Theorem 4.1. Let $\alpha \in L^{\infty}(\Omega), \beta \in L^{\infty}(\Gamma)$ be two non-negative, non-zero functions and let $f: \mathbb{R} \rightarrow \mathbb{R}$ be a locally essentially bounded function satisfying the subcritical growth in (4.1). Put $F(\xi)=\int_{0}^{\xi} f(t) d t$ for all $\xi \in \mathbb{R}$ and suppose that

(H1) $K_{1} \frac{1}{a_{1}^{p-1}}+K_{2} a_{1}^{s-p}<\frac{p\|\alpha\|_{L^{1}(\Omega)}}{\|q\|_{L^{1}(\Omega)}} \frac{\left[-F\left(a_{2}\right)\right]}{a_{2}^{p}}$ with positive constants $a_{1}, a_{2}$ satisfying $a_{1}<\left(\frac{\|q\|_{L^{1}(\Omega)}}{p}\right)^{\frac{1}{p}} a_{2}$ and $a_{2} \in K$;

(H2) $\limsup _{|\xi| \rightarrow+\infty} \frac{[-F(\xi)]}{|\xi|^{p}} \leq 0$.

Then, for each $\lambda \in \tilde{\lambda}$ and for each locally essentially bounded function $g: \mathbb{R} \rightarrow \mathbb{R}$ whose potential $G(\xi)=\int_{0}^{\xi} g(t) d t, \xi \in \mathbb{R}$, fulfills

(H3) $[-G(\xi)] \leq b_{3}|\xi|^{p}$ for all $\xi \in \mathbb{R}$ with $b_{3}$ being non-negative,

there exists $\bar{\delta}$ stated in (4.5) such that, for each $\mu \in[0, \bar{\delta}[$, problem (1.1) possesses at least three distinct solutions.

Proof. Similar to the proof of Theorem 3.1 we are going to apply Theorem 2.1 getting the assertion. Let $\lambda, \mu$ and $g$ be fixed satisfying the assumptions and as before, let $X:=W^{1, p}(\Omega)$ be endowed with the norm $\|\cdot\|_{W^{1, p}(\Omega)}$ given in (2.9). Now, we set again, for any $u \in X$,

$$
\begin{aligned}
& \Phi(u):=\frac{1}{p}\|u\|_{W^{1, p}(\Omega)}^{p}, \quad j(u)= \begin{cases}0, & \text { if } u \in K, \\
+\infty, & \text { otherwise },\end{cases} \\
& \Upsilon(u)=\int_{\Omega} \alpha(x)[-F(u(x))] d x+\frac{\mu}{\lambda} \int_{\Gamma} \beta(x)[-G(\gamma u(x)] d \sigma, \\
& \Psi(u):=\Upsilon(u)-j(u), \quad J_{\lambda}(u):=\Phi(u)-\lambda \Psi(u)
\end{aligned}
$$

leading to

$$
\begin{aligned}
& J_{\lambda}(u) \\
& =\frac{1}{p}\|u\|_{W^{1, p}(\Omega)}^{p}-\lambda \int_{\Omega} \alpha(x)[-F(u(x))] d x-\mu \int_{\Gamma} \beta(x)[-G(\gamma u(x)] d \sigma+\lambda j(u) .
\end{aligned}
$$

First, we observe that

$$
\varphi_{1}(r) \leq \frac{\sup _{\Phi(u)<r} \Upsilon(u)}{r}
$$


With the aid of assumption (H3) along with the subcritical growth on $f$ and the embeddings in (4.2), we may estimate $\Upsilon$ through

$$
\begin{aligned}
& \Upsilon(u) \\
& =\int_{\Omega} \alpha(x)[-F(u(x))] d x+\frac{\mu}{\lambda} \int_{\Gamma} \beta(x)[-G(\gamma u(x)] d \sigma \\
& \leq\|\alpha\|_{L^{\infty}(\Omega)} b_{1}\|u\|_{L^{1}(\Omega)}+\|\alpha\|_{L^{\infty}(\Omega)} \frac{b_{2}}{s}\|u\|_{L^{s}(\Omega)}^{s}+\frac{\mu}{\lambda}\|\beta\|_{L^{\infty}(\Gamma)} b_{3}\|\gamma u\|_{L^{p}(\Gamma)}^{p} \\
& \leq\|\alpha\|_{L^{\infty}(\Omega)} b_{1} c_{1}\|u\|_{W^{1, p}(\Omega)}+\|\alpha\|_{L^{\infty}(\Omega)} \frac{b_{2}}{s} c_{s}^{s}\|u\|_{W^{1, p}(\Omega)}^{s} \\
& \quad+\frac{\mu}{\lambda}\|\beta\|_{L^{\infty}(\Gamma)} b_{3} C^{p}\|u\|_{W^{1, p}(\Omega)}^{p} \\
& \leq \frac{K_{1}}{p^{\frac{1}{p}}}\|u\|_{W^{1, p}(\Omega)}+\frac{K_{2}}{p^{\frac{s}{p}}}\|u\|_{W^{1, p}(\Omega)}^{s}+\frac{\mu}{\lambda}\|\beta\|_{L^{\infty}(\Gamma)} b_{3} C^{p}\|u\|_{W^{1, p}(\Omega)}^{p} .
\end{aligned}
$$

Since $\Phi(u)<r$ is equivalent to $\|u\|_{W^{1, p}(\Omega)}<(p r)^{\frac{1}{p}}$, we obtain, by setting $r=a_{1}^{p}$,

$$
\begin{aligned}
\varphi_{1}(r) & \leq \frac{K_{1} r^{\frac{1}{p}}+K_{2} r^{\frac{s}{p}}+\frac{\mu}{\lambda}\|\beta\|_{L^{\infty}(\Gamma)} b_{3} C^{p} r p}{r} \\
& =K_{1} \frac{1}{a_{1}^{p-1}}+K_{2} a_{1}^{s-p}+\frac{\mu}{\lambda}\|\beta\|_{L^{\infty}(\Gamma)} b_{3} C^{p} p .
\end{aligned}
$$

On the other hand, we have

$$
\frac{\Psi\left(a_{2}\right)}{\Phi\left(a_{2}\right)} \geq \frac{p\|\alpha\|_{L^{1}(\Omega)}}{\|q\|_{L^{1}(\Omega)}} \frac{\left[-F\left(a_{2}\right)\right]}{a_{2}^{p}}+\frac{p\|\beta\|_{L^{1}(\Gamma)}}{\|q\|_{L^{1}(\Omega)}} \frac{\mu}{\lambda} \frac{\min \left\{0,-G\left(a_{2}\right)\right\}}{a_{2}^{p}} .
$$

Then, since $\mu<\delta$, there holds

$$
\mu<\frac{1-\lambda\left(K_{1} \frac{1}{a_{1}^{p-1}}+K_{2} a_{1}^{s-p}\right)}{\|\beta\|_{L^{\infty}(\Gamma)} b_{3} C^{p} p} \text { and } \quad \mu<\frac{1-\lambda\left(\frac{p\|\alpha\|_{L^{1}(\Omega)}}{\|q\|_{L^{1}(\Omega)}} \frac{\left[-F\left(a_{2}\right)\right]}{a_{2}^{p}}\right)}{\frac{p\|\beta\|_{L^{1}(\Gamma)}}{\|q\|_{L^{1}(\Omega)}} \frac{\min \left\{0,-G\left(a_{2}\right)\right\}}{a_{2}^{p}}} .
$$

This yields

$$
K_{1} \frac{1}{a_{1}^{p-1}}+K_{2} a_{1}^{s-p}+\frac{\mu}{\lambda}\|\beta\|_{L^{\infty}(\Gamma)} b_{3} C^{p} p<\frac{1}{\lambda}
$$

and

$$
\frac{p\|\alpha\|_{L^{1}(\Omega)}}{\|q\|_{L^{1}(\Omega)}} \frac{\left[-F\left(a_{2}\right)\right]}{a_{2}^{p}}+\frac{p\|\beta\|_{L^{1}(\Gamma)}}{\|q\|_{L^{1}(\Omega)}} \frac{\mu}{\lambda} \frac{\min \left\{0,-G\left(a_{2}\right)\right\}}{a_{2}^{p}}>\frac{1}{\lambda} .
$$

Finally, from the estimates above, we conclude

$$
\varphi_{1}(r)<\frac{1}{\lambda}<\frac{\Psi\left(a_{2}\right)}{\Phi\left(a_{2}\right)} .
$$

As already mentioned in the proof of Theorem 3.1, it holds, in particular,

$$
\frac{\sup _{\Phi(x)<r} \Psi(x)}{r} \leq \frac{\sup _{\Phi(x) \leq r} \Psi(x)}{r}<\frac{\Psi\left(a_{2}\right)}{\Phi\left(a_{2}\right)}, \quad 0<r<\Phi\left(a_{2}\right) .
$$


Thus, we derive

$$
\varphi_{2}(r) \geq \frac{\Psi\left(a_{2}\right)-\sup _{\Phi(x) \leq r} \Psi(x)}{\Phi\left(a_{2}\right)-r} \geq \frac{\Psi\left(a_{2}\right)-r \frac{\Psi\left(a_{2}\right)}{\Phi\left(a_{2}\right)}}{\Phi\left(a_{2}\right)-r}=\frac{\Psi\left(a_{2}\right)}{\Phi\left(a_{2}\right)} .
$$

Combining these estimates yields

$$
\varphi_{1}(r)<\frac{1}{\lambda}<\varphi_{2}(r)
$$

Finally, we note that the functional $J_{\lambda}$ is coercive and satisfies the Palais-Smale condition (cf. the proof of Theorem 3.1). Hence, the assumptions of Theorem 2.1 are satisfied which ensures the existence of three distinct critical points of $J_{\lambda}$. That these critical points are solutions of (1.1) can be shown using the same arguments as in the end of the proof of Theorem 3.1. That finishes the proof of the theorem.

We conclude with an application of Theorem 4.1.

Example 4.2. Let $m$ be a positive constant specified later and let $s \in] 1, \frac{N p}{N-p}[$ if $p<N$ as well as $s \in] 1,+\infty[$ if $p=N$. We define the functions $f: \mathbb{R} \rightarrow \mathbb{R}$ and $g: \mathbb{R} \rightarrow \mathbb{R}$ through

$$
\begin{aligned}
& f(t)= \begin{cases}-\tilde{C} & \text { if } t<0, \\
-1-|t|^{s-1} & \text { if } 0 \leq t \leq m, \\
-\frac{\left(1+m^{2}\right)\left(1+m^{s-1}\right)}{1+t^{2}} & \text { if } t>m,\end{cases} \\
& g(t)= \begin{cases}-|t|^{p-1} & \text { if } t \leq 1, \\
-2|t|^{p-1} & \text { if } t>1,\end{cases}
\end{aligned}
$$

with $\tilde{C}$ being a positive constant. Hence, for every constant $m>0$, the mapping $f$ fulfills the subcritical growth in (4.1) with $b_{1}=\max \{1, \tilde{C}\}$ and $b_{2}=1$. With the aid of these constants we define the numbers $K_{1}$ and $K_{2}$ as in (4.3). Now, fix m such that

$$
m>\max \left\{\left(\frac{p}{\|q\|_{L^{1}(\Omega)}}\right)^{\frac{1}{p}},\left(s \frac{K_{1}+K_{2}}{p} \frac{\|q\|_{L^{1}(\Omega)}}{\|\alpha\|_{L^{1}(\Omega)}}\right)^{\frac{1}{s-p}}\right\} .
$$

Thanks to condition (4.7) it is clear that inequality

$$
1<\left(\frac{\|q\|_{L^{1}(\Omega)}}{p}\right)^{\frac{1}{p}} m
$$

holds true. Moreover, we have

$$
\frac{-F(m)}{m^{p}}=\frac{m^{s-p}}{s}+\frac{1}{m^{p-1}}>\frac{K_{1}+K_{2}}{p} \frac{\|q\|_{L^{1}(\Omega)}}{\|\alpha\|_{L^{1}(\Omega)}} .
$$

Setting $a_{1}=1$ and $a_{2}=m$ we see at once that condition (H1) in Theorem 4.1 is satisfied. Additionally, for $\xi_{1}>m$ and $\xi_{2}<0$, we point out that

$$
\begin{aligned}
& \frac{\left[-F\left(\xi_{1}\right)\right]}{\left|\xi_{1}\right|^{p}}=\left(1+m^{2}\right)\left(1+m^{s-1}\right) \frac{\arctan \left(\xi_{1}\right)}{\left|\xi_{1}\right|^{p}} \rightarrow 0 \text { as } \xi_{1} \rightarrow+\infty, \\
& \frac{\left[-F\left(\xi_{2}\right)\right]}{\left|\xi_{2}\right|^{p}}=\tilde{C} \frac{\left|\xi_{2}\right|}{\left|\xi_{2}\right|^{p}} \rightarrow 0 \text { as } \xi_{2} \rightarrow-\infty
\end{aligned}
$$


ensuring that (H2) is fulfilled as well. Since assumption (H3) holds for $b_{3}=\frac{2}{p}$, Theorem 4.1 can be applied to problem (1.1) with the special data in (4.6) which yields the existence of three distinct solutions of (1.1).

\section{ACKNOWLEDGEMENTS}

The authors wish to thank Prof. D. Motreanu for his encouragement in writing this work.

\section{REFERENCES}

[1] D. Averna, G. Bonanno, Three solutions for a Neumann boundary value problem involving the p-Laplacian, Matematiche (Catania) 60 (2005), no. 1, 81-91.

[2] G. Barletta, Existence results for semilinear elliptical hemivariational inequalities, Nonlinear Anal. 68 (2008), no. 8, 2417-2430.

[3] G. Bonanno, P. Candito, Non-differentiable functionals and applications to elliptic problems with discontinuous nonlinearities, J. Differential Equations 244 (2008), no. 12, 3031-3059.

[4] G. Bonanno, P. Candito, On a class of nonlinear variational-hemivariational inequalities, Appl. Anal. 83 (2004), no. 12, 1229-1244.

[5] G. Bonanno, P. Candito, Three solutions to a Neumann problem for elliptic equations involving the p-Laplacian, Arch. Math. (Basel) 80 (2003), no. 4, 424-429.

[6] G. Bonanno, G. D'Aguì, Multiplicity results for a perturbed elliptic Neumann problem, Abstr. Appl. Anal. vol. 2010, Art. ID 564363, 10 pages, 2010.

[7] G. Bonanno, G. D'Aguì, On the Neumann problem for elliptic equations involving the pLaplacian, J. Math. Anal. Appl. 358 (2009), no. 2, 223-228.

[8] G. Bonanno, S. A. Marano, On the structure of the critical set of non-differentiable functions with a weak compactness condition, Appl. Anal. 89 (2010), no. 1, 1-10.

[9] G. Bonanno, D. Motreanu, P. Winkert, Variational-hemivariational inequalities with small perturbations of nonhomogeneous Neumann boundary conditions, J. Math. Anal. Appl. 381 (2011), no. 2, 627-637.

[10] H. Brezis, Analyse fonctionnelle, Masson, Paris, 1983.

[11] P. Candito, Infinitely many solutions to the Neumann problem for elliptic equations involving the p-Laplacian and with discontinuous nonlinearities, Proc. Edinb. Math. Soc. (2) 45 (2002), 397-409.

[12] S. Carl, Equivalence of some multi-valued elliptic variational inequalities and variationalhemivariational inequalities, Adv. Nonlinear Stud. 11 (2011), no. 2, 247-263.

[13] S. Carl, Parameter-dependent variational-hemivariational inequalities and an unstable degenerate elliptic free boundary problem, Nonlinear Anal. Real World Appl. 12 (2011), no. 6, $3185-3198$.

[14] S. Carl, V. K. Le, D. Motreanu, Nonsmooth variational problems and their inequalities, Springer Monographs in Mathematics, Springer, New York, New York, 2007.

[15] S. Carl, Z. Naniewicz, Vector quasi-hemivariational inequalities and discontinuous elliptic systems, J. Global Optim. 34 (2006), no. 4, 609-634.

[16] S. Carl, P. Winkert, General comparison principle for variational-hemivariational inequalities, J. Inequal. Appl. vol. 2009, Art. ID 184348, 29 pages, 2009.

[17] K. C. Chang, Variational methods for nondifferentiable functionals and their applications to partial differential equations, J. Math. Anal. Appl. 80 (1981), 102-129.

[18] F. H. Clarke, Optimization and Nonsmooth Analysis, Classics in Applied Mathematics, vol. 5, SIAM, Philadelphia, 1990.

[19] G. D'Aguì, G. Molica Bisci, Three non-zero solutions for elliptic Neumann problems, Anal. Appl. (Singap.) 9 (2011), no. 4, 383-394.

[20] J. Fernández Bonder, J. D. Rossi, Existence results for the $p$-Laplacian with nonlinear boundary conditions, J. Math. Anal. Appl. (2001), no. 1, 195-223.

[21] J. Fernández Bonder, Multiple positive solutions for quasilinear elliptic problems with signchanging nonlinearities, Abstr. Appl. Anal. vol. 2004, no. 12, 1047-1055, 2004.

[22] J. Fernández Bonder, Multiple solutions for the p-Laplace equation with nonlinear boundary conditions, Electron. J. Differential Equations, no. 37, 7 pages, 2006. 
[23] M. E. Filippakis, N. S. Papageorgiou, Solvability of nonlinear variational-hemivariational inequalities, J. Math. Anal. Appl. 311 (2005), no. 1, 162-181.

[24] D. Goeleven, D. Motreanu, P. D. Panagiotopoulos, Eigenvalue problems for variationalhemivariational inequalities at resonance, Nonlinear Anal. 33 (1998), no. 2, 161-180.

[25] D. Kinderlehrer, An introduction to variational inequalities and their applications, Pure and Applied Mathematics, vol. 88, Academic Press, New York, 1980.

[26] A. Kristály, G. Moroşanu, New competition phenomena in Dirichlet problems, J. Math. Pures Appl. 94 (2010), 555-570.

[27] A. Kristály, D. Motreanu, Nonsmooth Neumann-type problems involving the p-Laplacian, Numer. Funct. Anal. Optim. 28 (2007), 1309-1326.

[28] A. Kristály, C. Varga, V. Varga, A nonsmooth principle of symmetric criticality and variational-hemivariational inequalities, J. Math. Anal. Appl. 325 (2007), no. 2, 975-986.

[29] S. Th. Kyritsi, N. S. Papageorgiou, Nonsmooth critical point theory on closed convex sets and nonlinear hemivariational inequalities, Nonlinear Anal. 61 (2005), no. 3, 373-403.

[30] C. Li, S. Li, Multiple solutions and sign-changing solutions of a class of nonlinear elliptic equations with Neumann boundary condition, J. Math. Anal. Appl. 298 (2004), no. 1, 14-32.

[31] H. Lisei, C. Varga, Multiple solutions for a differential inclusion problem with nonhomogeneous boundary conditions, Numer. Funct. Anal. Optim. 30 (2009), no. 5-6, 566-581.

[32] C. Liu, Y. Zheng, Linking solutions for p-Laplace equations with nonlinear boundary conditions and indefinite weight, Calc. Var. Partial Differential Equations 41 (2011), no. 1-2, 261-284.

[33] R. Livrea and S.A. Marano, Existence and classification of critical points for nondifferentiable functions, Adv. Differential Equations 9 (2004), 961-978.

[34] S. A. Marano, G. Molica Bisci, D. Motreanu, Multiple solutions for a class of elliptic hemivariational inequalities, J. Math. Anal. Appl. 337 (2008), no. 1, 85-97.

[35] S. A. Marano, D. Motreanu, On a three critical points theorem for non-differentiable functions and applications to nonlinear boundary value problems, Nonlinear Anal. 48 (2002), no. 1, 3752 .

[36] S. A. Marano, N. S. Papageorgiou, On some elliptic hemivariational and variationalhemivariational inequalities, Nonlinear Anal. 62 (2005), no. 4, 757-774.

[37] S. R. Martínez, J. D. Rossi, Weak solutions for the p-Laplacian with a nonlinear boundary condition at resonance, Electron. J. Differential Equations, no. 27, 14 pages, 2003.

[38] V. G. Maz'ja, Sobolev spaces, Springer Series in Soviet Mathematics, Springer-Verlag, Berlin, 1985.

[39] D. Motreanu, P. D. Panagiotopoulos, Minimax Theorems and Qualitative Properties of the Solutions of Hemivariational Inequalities, Nonconvex Optim. Appl. 29 Kluwer, Dordrecht, 1998.

[40] D. Motreanu, V. Rădulescu, Variational and Non-variational Methods in Nonlinear Analysis and Boundary Value Problems, Nonconvex Optimization and Applications, Kluwer Academic Publishers 2003.

[41] D. Motreanu, P. Winkert, Variational-hemivariational inequalities with nonhomogeneous Neumann boundary condition, Matematiche (Catania) 65 (2010), no. 2, 109-119.

[42] Z. Naniewicz, P. D. Panagiotopoulos, Mathematical theory of hemivariational inequalities and applications, Monographs and Textbooks in Pure and Applied Mathematics, vol. 188, Marcel Dekker Inc., New York, 1995.

[43] P. D. Panagiotopoulos, Hemivariational inequalities, Applications in mechanics and engineering, Springer-Verlag, Berlin, 1993.

[44] B. Ricceri, A further refinement of a three critical points theorem, Nonlinear Anal. 74 (2011), no. $18,7446-7454$

[45] M. Struwe, Variational methods, Springer-Verlag, Berlin, 1996.

[46] P. Winkert, Constant-sign and sign-changing solutions for nonlinear elliptic equations with Neumann boundary values, Adv. Differential Equations 15 (2010), no. 5-6, 561-599.

[47] P. Winkert, Multiple solution results for elliptic Neumann problems involving set-valued nonlinearities, J. Math. Anal. Appl. 377 (2011), no. 1, 121-134.

[48] J.-H. Zhao, P.-H. Zhao, Existence of infinitely many weak solutions for the p-Laplacian with nonlinear boundary conditions, Nonlinear Anal. 69 (2008), no. 4, 1343-1355. 
(G. Bonanno) Department of Civil, Computer, Construction, Environmental Engineering and Applied Mathematics, University of Messina, 98166 Messina, Italy.

E-mail address: bonanno@unime.it

(P. Winkert) Technische Universität Berlin, Institut für Mathematik, Strasse des 17. Juni 136, 10623 Berlin, Germany.

E-mail address: winkert@math.tu-berlin.de 